\title{
Chronic Candidiasis of Tongue with kissing Lesion on the Palate in HIV Positive Patient
}

\author{
${ }^{1}$ Gargi S Sarode, ${ }^{2}$ Sachin C Sarode, ${ }^{3}$ Shankargouda Patil
}

\begin{abstract}
Candidiasis is the most common oral opportunistic infection affecting people with acquired immunodeficiency. Here, in this case report, we are presenting a case of an HIV positive patient with chronic pseudomembranous candidiasis on tongue along with a kissing lesion on the palate. This case report emphasizes that the aforementioned finding can serve as a good clinical marker for prediction and early detection of HIV infection.
\end{abstract}

Keywords: Candidiasis, Palate, HIV positive.

How to cite this article: Sarode GS, Sarode SC, Patil S. Chronic Candidiasis of Tongue with kissing Lesion on the Palate in HIV Positive Patient. World J Dent 2014;5(4):247-248.

Source of support: Nil

\section{Conflict of interest: None}

The most common oral opportunistic infection affecting persons with acquired immunodeficiency is candidiasis. This article reports an candidal infection of the tongue with candidal infection of the palate together in the patients who have the human immunodeficiency viral infection. A retrospective analysis of 336 patients with acquired immunodeficiency syndrome revealed a prevalence of $8 \%$ with candidat infection of both the tongue and palate. Patients in this group were significantly older (39.2 vs 34.5 years) and had significantly lower CD4-CD8 ratios than did the rest of the cohort.

Among all the oral sites, tongue is the most common location for development of candidiasis in dentulous patients. In contrast, palatal candidiasis is comparatively less common in dentulous patient. But chronic candidiasis of tongue along with a simultaneous palatal lesion (kissing lesion) could suggest some serious underlying predisposing pathology.

\footnotetext{
${ }^{1-3}$ Associate Professor

1,2Department of Oral Pathology and Microbiology, Dr DY Patil Dental College and Hospital, Pune, Maharashtra, India

${ }^{3}$ Department of Oral Pathology and Microbiology, Faculty of Dentistry, MS Ramaiah University of Applied Sciences Bengaluru, Karnataka, India

Corresponding Author: Sachin C Sarode, Associate Professor Department of Oral Pathology and Microbiology, Dr DY Patil Dental College and Hospital, Maheshnagar, Pimpri, Pune-411018 Maharashtra, India, Phone: +919922491465, e-mail: drsachin sarode@gmail.com
}

A 37-year-old male patient reported with soreness of the oral cavity since 1 month. His medical and dental history was unremarkable. Intraoral examination revealed focal white scrapable patches on tongue distributed homogeneously through out the dorsal surface. At places, depapillation and erythematous areas were observed (Fig. 1). Examination of palate showed bilateral erythematous area (Fig. 2). Smear was taken from the tongue and the palatal lesion, which showed predominantly candidal hyphae, and mildly dysplastic exfoliated epithelial cells. Many candida hyphae were seen to invade the epithelial cells. Based on the clinical presentation and cytology, diagnosis of chronic pseudomembranous candidiasis was made. Further immunosuppression and antibiotic therapy related history of patient was not conclusive. Hematological parameters were within normal limits. Patient was advised investigations to rule out HIV infection. Patient was diagnosed HIV positive by ELISA test with CD4 count of 93 . Viral load was 38,000 copies $/ \mathrm{ml}$. The patient was treated with highly active antiretroviral therapy with did anosine (100 $\mathrm{mg}$ twice daily), stavudine (40 mg twice daily), and ritonavir (600 mg twice daily), for the HIV infection. For candidiasis, topical antifungal was prescribed. After therapy, viral load decreased and oral lesions regressed.

A retrospective analysis of 336 patients with acquired immunodeficiency syndrome revealed a prevalence of $8 \%$

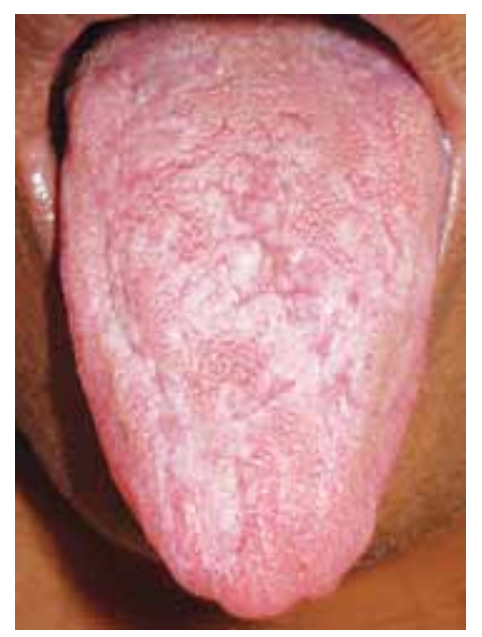

Fig. 1: Intraoral photograph showing focal areas of white scrapable patches on dorsal surface of the tongue 


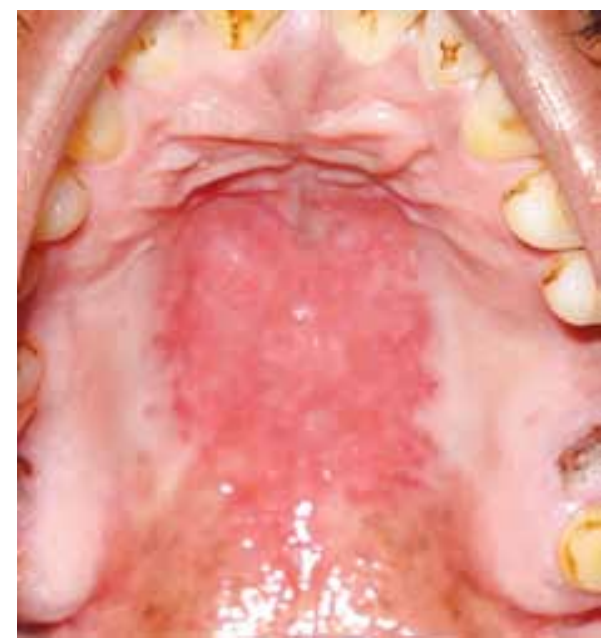

Fig. 2: Intraoral photograph showing erythematous area on the palate

with candidal infection of both the tongue and palate. Such patients are significantly older (39.2 vs 34.5 years) and have significantly lower CD4-CD8 ratio. ${ }^{1}$
HIV infection is a major health problem in India. The National AIDS Control Organization (NACO) of India reports a seropositivity of 25.03 per thousand for the whole country, as of October, $1999 .^{2}$ Early diagnosis and treatment plays a very important role in the prognosis of HIV positive patients. This case report emphasizes the finding that candidiasis of tongue along with the kissing lesion of palate could serve as a good clinical marker for prediction and early detection of HIV infection.

\section{REFERENCES}

1. Touyz L, Harel-Raviv M, Prosterman B, Gornitsky M. Candida infection of the tongue together with candidal infection of the palate in patients with the human immunodeficiency virus. Quintessence Int 1997;27(2):89-92.

2. Ranganathan K, Reddy BV, Kumarasamy N, Solomon S, Viswanathan R, Johnson NW. Oral lesions and conditions associated with human immunodeficiency virus infection in 300 south Indian patients. Oral Diseases 2000;6(3): 152-157. 